\title{
Electron-electron versus electron-phonon interactions in lattice models: Screening effects described by a density functional theory approach
}

\author{
E. Viñas Boström, ${ }^{1}$ P. Helmer, ${ }^{1}$ P. Werner, ${ }^{2}$ and C. Verdozzi ${ }^{1}$ \\ ${ }^{1}$ Department of Physics and ETSF, Lund University, P.O. Box 118, 22100 Lund, Sweden \\ ${ }^{2}$ Department of Physics, University of Fribourg, 1700 Fribourg, Switzerland
}

(Received 6 March 2019; revised manuscript received 12 August 2019; published 29 August 2019)

\begin{abstract}
We address the interplay of electron-electron $(e-e)$ and electron-phonon ( $e$-ph) interactions in the HubbardHolstein model, using a two-component density functional theory. Exchange-correlation potentials constructed via dynamical mean field theory for a $D=\infty$ Bethe lattice and analytically for an isolated site give a new perspective on $e$-ph screening of the $e$ - $e$ interactions and its effect on the charge- and spin-Kondo regimes. Comparisons to exact benchmarks show that the approach is suitable to describe transport properties and realtime dynamics in homogeneous and inhomogeneous lattice systems.
\end{abstract}

DOI: 10.1103/PhysRevResearch.1.013017

The quantum theory of solids, now almost a century old, is unquestionably a great success story: A vast number of material properties and phenomena have been explained, resulting in technological advances with transformative effects $[1,2]$. Many questions, however, remain unresolved or, since the frontier of knowledge progresses, require a deeper level of understanding. One of these is the long-standing problem of how to describe systems where the effects of electron-electron $(e-e)$ and electron-phonon ( $e$-ph) interactions are equally important [3]. For such systems, key requisites of a theoretical approach are a proper treatment of the dynamical screening of $e-e$ interactions due to phonons and the possibility of dealing in a feasible way with inhomogeneous situations, e.g., chemically doped solids or systems with applied (time- and space-dependent) external fields. Although highly challenging to describe, such systems are of growing interest because of the expectation that they may host novel physical phenomena and phases, with unexplored functionalities $[4,5]$.

At this stage, even simple model systems can provide important insight into these aspects. A minimum-complexity template that accounts on equal footing for $e-e$ and $e$-ph interactions in a lattice is the Hubbard-Holstein $(\mathrm{HH})$ model [6-16]. Depending on model parameters and dimensionality $D$, it displays a broad range of interesting behaviors, e.g., metal-insulator transitions [11], bipolaronic phases [12], superconductivity, and charge density order [10]. Out of equilibrium, it has been used to study interaction quenches [17], dynamical insulator-to-metal transitions [18], and pump-probe dynamics $[19,20]$. Finally, for phonon overscreening of the

Published by the American Physical Society under the terms of the Creative Commons Attribution 4.0 International license. Further distribution of this work must maintain attribution to the author(s) and the published article's title, journal citation, and DOI. $e-e$ interactions, it motivates the study of lattice models with attractive Hubbard interactions [21-23].

Many approaches have been used to investigate the $\mathrm{HH}$ model [24], but so far neither density functional theory (DFT) $[25,26]$ nor its time-dependent generalization (TDDFT) [27] (they both play a central role in the study of realistic materials $[28,29]$ ), have been employed [30]. (TD)DFT can provide a new perspective on $e$-ph screening and is suitable for inhomogeneous systems. We thus consider here a (TD)DFT approach to the $\mathrm{HH}$ model. As for electron-nuclei systems in the continuum [31,32] or quantum electrodynamics [33,34], we use a two-component formulation. In our case, the basic variables for the electron (phonon) component are the electron occupations $\left\{n_{i}\right\}$ (phonon coordinates $\left\{x_{i}\right\}$ ) at each site $i$. Each component is governed by its own exchange-correlation (XC) potential. We explicitly determine the XC potentials for the analytically solvable one-site, zero-dimensional $(D=0)$ model, and the infinite-dimensional $(D=\infty)$ homogenous Bethe lattice (where $n_{i}=n, x_{i}=x$ ), via dynamical mean field theory (DMFT) [35-37]. These potentials are then used to address the dynamics of a finite system.

We find the following: (i) the behavior of the electronic $\mathrm{XC}$ potential is mainly determined by the phonon screened interaction $U^{\prime}$ and dynamical contributions to such screening are important. Furthermore, in the $\mathrm{HH}$ model the phonon $\mathrm{XC}$ potential is zero. (ii) The electronic XC potential is discontinuous at half-filling density $n=1$ for $U^{\prime}>0$ and at $n=0,2$ for $U^{\prime}<0$; for the Bethe lattice, the discontinuity appears above a nonzero value of $\left|U^{\prime}\right|$. (iii) For an infinite chain with a HH impurity, (TD)DFT conductances evolve smoothly from the charge- to the spin-Kondo regime upon varying the $e$-ph coupling. (iv) TDDFT dynamics in a test system subject to interaction quenches or external fields compares well with exact numerics in an appreciable range of interaction strengths. These results demonstrate that (TD)DFT is a promising formalism for the study of $e$-ph (inhomogeneous) lattice systems. 


\section{THE SYSTEM}

As starting point for the DFT description, we consider the inhomogeneous HH Hamiltonian

$$
\begin{aligned}
\hat{H}= & \sum_{i \sigma}\left(v_{i}-\mu\right) \hat{n}_{i \sigma}+U \sum_{i} \hat{n}_{i \uparrow} \hat{n}_{i \downarrow}-J \sum_{\langle i j\rangle \sigma} c_{i \sigma}^{\dagger} c_{j \sigma}+\omega \sum_{i} b_{i}^{\dagger} b_{i} \\
& +\sum_{i} \sqrt{2} \eta_{i} \hat{x}_{i}+\sqrt{2} g \sum_{i}\left(\hat{n}_{i \uparrow}+\hat{n}_{i \downarrow}-1\right) \hat{x}_{i},
\end{aligned}
$$

where $v_{i}$ is a local site-dependent electron potential, $\mu$ is the chemical potential, $U$ is the $e-e$ interaction strength, $J$ is the hopping amplitude (set equal to 1, as the energy unit), and $\langle\cdots\rangle$ denotes nearest neighbor sites. The operator $c_{i \sigma}^{\dagger}$ creates an electron on site $i$ with spin $\sigma$, with $\hat{n}_{i \sigma}=c_{i \sigma}^{\dagger} c_{i \sigma}$ the corresponding density operator. The phonon frequency is $\omega$, and $g$ is the $e$-ph coupling. We use $\lambda=g^{2} / \omega$ as a measure of the $e$-ph interaction strength. Finally, the site-dependent external phonon potential $\eta_{i}$ is introduced to control the phonon coordinates $\hat{x}_{i}=\left(b_{i}^{\dagger}+b_{i}\right) / \sqrt{2}$, where $b_{i}$ destroys a phonon at site $i$. The form of $\hat{H}$ in Eq. (1) allows us to address formal aspects of the (TD)DFT description and to use a homogeneous $\mathrm{HH}$ reference system in (adiabatic) local density approximations. In particular, it allows us to prove the Hohenberg-Kohn theorem for the $\mathrm{HH}$ model (see Appendix A), thus putting the DFT treatment on solid ground.

To calculate the ground-state energy of the reference homogenous HH model, we perform the Lang-Firsov transformation $\hat{H} \rightarrow \hat{H}^{\prime}=e^{i \hat{S}} \hat{H} e^{-i \hat{S}}$ [38], resulting in

$$
\begin{aligned}
\hat{H}^{\prime}= & \sum_{i \sigma}\left(v_{i}^{\prime}-\mu\right) \hat{n}_{i \sigma}+\sum_{i} U^{\prime} \hat{n}_{i \uparrow} \hat{n}_{i \downarrow}-\sum_{\langle i j\rangle \sigma} \hat{J}_{i j}^{\prime} c_{i \sigma}^{\dagger} c_{j \sigma} \\
& +\sum_{i}\left[\omega b_{i}^{\dagger} b_{i}-\frac{\left(\eta_{i}-g\right)^{2}}{\omega}\right] .
\end{aligned}
$$

In $\hat{H}^{\prime}$, the hopping amplitude is renormalized as $J \rightarrow \hat{J}_{i j}^{\prime}=$ $J e^{i \sqrt{2} g\left(\hat{p}_{i}-\hat{p}_{j}\right) / \omega}$, with $\hat{p}_{i}=i\left(b_{i}^{\dagger}-b_{i}\right) / \sqrt{2}$ the phonon momentum, and the other parameters transform as $v \rightarrow v^{\prime}=v+$ $\left(g^{2}+2 g \eta\right) / \omega$, and $U \rightarrow U^{\prime}=U-2 g^{2} / \omega$.

\section{DENSITY FUNCTIONAL THEORY}

In addition to $a b$ initio descriptions of matter, (TD)DFT has been applied to model Hamiltonians to explore conceptual and methodological aspects of the theory [39-51], as well as for specific applications to cold atoms [52-55], Kondo physics [56-58], quantum transport [59-61], quantum electrodynamics [33], and nonequilibrium thermodynamics [62], to mention a few [63]. We here consider a two-component DFT for the HH model, where the basic variables are given by the set $(n, x) \equiv\left(\left\{n_{i}\right\},\left\{x_{i}\right\}\right)$, with $n_{i}=n_{i \uparrow}+n_{i \downarrow}$ being the total electron density at site $i$, and the conjugated fields are $(v, \eta) \equiv$ $\left(\left\{v_{i}\right\},\left\{\eta_{i}\right\}\right)$. In Appendix A, we prove that (i) the total energy $E=E[n, x]$ is a functional of $n$ and $x$, with a minimum at the ground-state values $\left(n_{0}, x_{0}\right)$ (the Hohenberg-Kohn theorem for the $\mathrm{HH}$ ) and (ii) where $v_{0}$ representability holds, $\left(n_{0}, x_{0}\right)$ is obtained by solving a two-component Kohn-Sham (KS) problem. In a homogeneous system (useful to derive a local density approximation), $n_{i}=n$ and $x_{i}=x$ for all $i$, and the
KS Hamiltonian is $H_{K S}=H_{s}^{(e)}+H_{s}^{(\mathrm{ph})}$, with

$$
\begin{aligned}
H_{s}^{(e)} & =\left(v_{K S}[n, x]-\mu\right) \sum_{i \sigma} \hat{n}_{i \sigma}-\sum_{\langle i j\rangle \sigma} J\left(c_{i \sigma}^{\dagger} c_{j \sigma}+\text { H.c. }\right), \\
H_{s}^{(\mathrm{ph})} & =\omega \sum_{i} b_{i}^{\dagger} b_{i}+\sqrt{2} \eta_{K S}[n, x] \sum_{i} \hat{x}_{i} .
\end{aligned}
$$

The electronic KS potential can be written as $v_{K S}=v_{\mathrm{ext}}+$ $v_{H x c}$, with $v_{\mathrm{ext}} \equiv v$ and the Hartree-exchange-correlation (Hxc) part $v_{H x c}=U n / 2+\sqrt{2} g x+\delta E_{x c} / \delta n$. To obtain $E_{x c}$, we subtract from $E[n, x]$ the $e$-e and $e$-ph Hartree interaction terms, and the energies of the corresponding noninteracting HH system. For the phonons, $\eta_{K S}=\eta_{\text {ext }}+\eta_{H x c}$, where $\eta_{\text {ext }} \equiv$ $\eta$ and $\eta_{H x c} \equiv \eta_{H}+\eta_{x c}=g(n-1)+\delta E_{x c} / \delta x$.

Below we consider two different approximations to the electronic exchange-correlation potential: The first is given by the exact potential of a single $\mathrm{HH}$ site, while the second is constructed from a dynamical mean-field theory (DMFT) solution of the $\mathrm{HH}$ model on the infinitely coordinated Bethe lattice. To obtain the phonon exchange-correlation potential, we note that it follows from the Heisenberg equation of motion $\partial_{t} p=i \hbar^{-1}[H, p]=0$ for the phonon momentum that $x=-\sqrt{2}[g(n-1)+\eta] / \omega$. Inverting this relation gives the external potential $\eta_{\text {ext }}=-g(n-1)-\omega x / \sqrt{2}$. Since the phonon Kohn-Sham potential $\eta_{K S}$ is defined to reproduce the interacting value of $x$ in a noninteracting system, it is obtained by taking $g=0$ in the previous equation. We thus find $\eta_{H x c}=$ $\eta_{K S}-\eta_{\mathrm{ext}}=g(n-1)$, showing that $\eta_{H x c}=\eta_{H}$ and $\eta_{x c}=0$. This argument is independent of dimensionality and it holds in all cases considered below.

\section{A. $v_{x c}$ from a single Hubbard-Holstein site}

We consider a single HH site exchanging energy and particles with a bath at chemical potential $\mu$ and temperature $\beta^{-1}$. This problem is analytically solvable by determining the partition function $Z=\operatorname{Tr} e^{-\beta H(v, \eta)}$ (see Appendix B for further details). Using $n=-\beta^{-1} \partial_{v} \ln Z, x=-(\sqrt{2} \beta)^{-1} \partial_{\eta} \ln Z$, and solving for $(v, \eta)$, we find

$$
v_{x c}(n, x)=(1-\delta n) \frac{U^{\prime}}{2}+\frac{g^{2}}{\omega} \delta n+\frac{1}{\beta} \ln \frac{\delta n+R}{1+\delta n},
$$

where $\delta n=n-1, R=\left[e^{-\beta U^{\prime}}\left(1-\delta n^{2}\right)+\delta n^{2}\right]^{1 / 2}$, and $U^{\prime}$ defined above. Thus, $v_{x c}$ is independent of $x$ and depends on the phonon screened $e$-e interaction. For $g=0$, we recover the expression for a single-site Hubbard system [56]. In Fig. 1(a), we display $v_{x c}$ as a function of $n$ and $\lambda$ for $U=1$ and $\beta=$ 20. For $U^{\prime}>0$ (i.e., $\lambda<1 / 2$ ) the potential is discontinuous at $n=1$, as in the case of the purely electronic repulsive Hubbard model $[40,56,64,65]$. For $U^{\prime}<0$ (i.e., $\lambda>1 / 2$ ), there are discontinuities at $n=0$ and $n=2$, as in a negative- $U$ Hubbard model [23,53]. Notably, in the present model, the transition from positive to negative $U^{\prime}$ results from phonon screening of the e-e interaction. Equation (4) shows that, save for the linear term $g^{2} \delta n / \omega$, the analytic expressions for $v_{x c}$ in a $\mathrm{HH}$ and a Hubbard single-site model only differ by the renormalization $U \rightarrow U^{\prime}$; i.e., $e$-ph interactions primarily affect the discontinuities at $n=\{0,1,2\}$. 


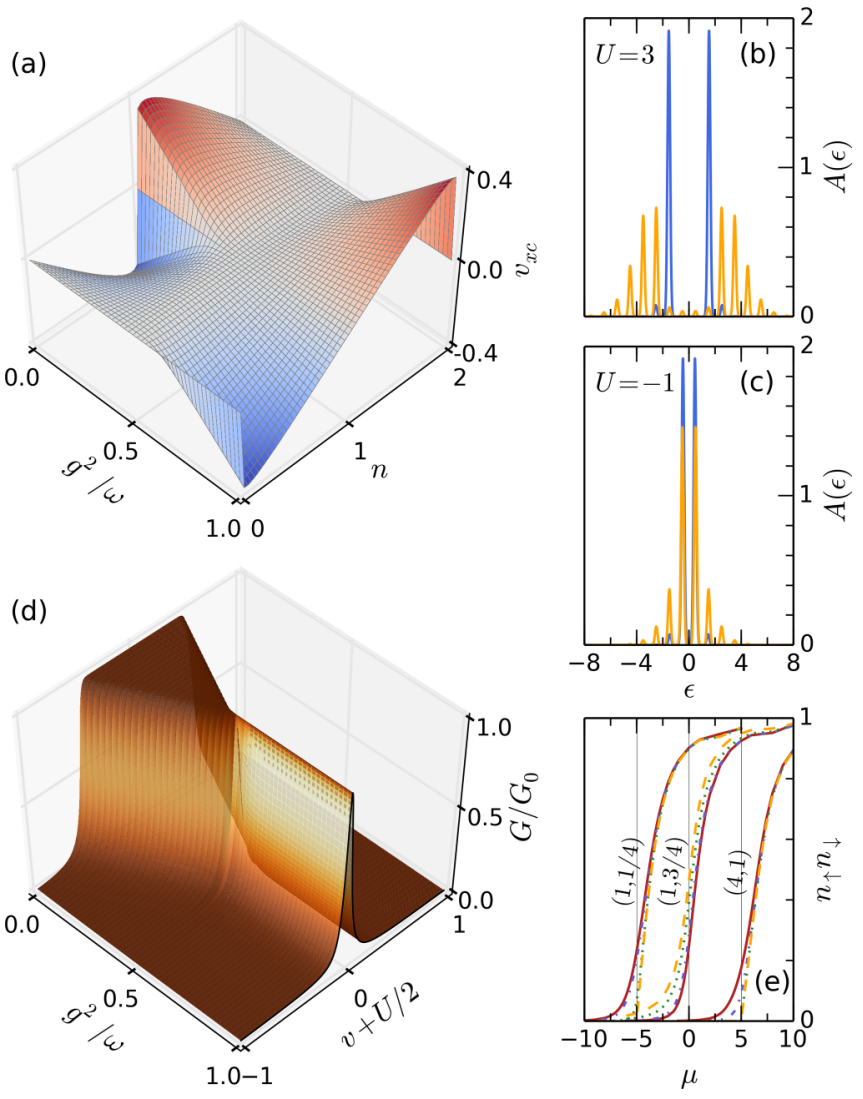

FIG. 1. Properties of a single Hubbard-Holstein site: (a) Exchange-correlation potential $v_{x c}$ for $U=1$ and $\beta=20$ as a function of $n$ and $\lambda=g^{2} / \omega$. [(b), (c)] Spectral function $A(\epsilon)$ for $\mu=0$, $v=-U / 2$, and $\beta=5$. The blue curves are for $\lambda=0.08$ and yellow curves for $\lambda=1$. (d) Zero bias conductance $G$ for $U=1, \mu=0$, and $\beta \rightarrow \infty$, as a function of $v$ and $\lambda$. (e) Double occupancy $\left\langle n_{\uparrow} n_{\downarrow}\right\rangle$ as a function of chemical potential $\mu$. The three sets of curves pertain to different $(U, g)$ pairs as indicated, and $v=-U / 2$. For readability, the leftmost (rightmost) set of curves is shifted by $-5(+5)$ along the abscissa. In each set, the vertical thin line refers to $\mu=0$, the red curve represents the exact result for $\beta=\infty$, while the DFT ones, obtained with $\mathcal{D}^{s s}$ as in Eq. (5), are for $\beta=1$ (blue), $\beta=5$ (green), and $\beta=10$ (yellow). In all panels, $\omega=1$ and $\eta=0$.

Phonon effects are instead explicitly manifest in the electronic spectral function $A(\epsilon)$. Starting from the many-body Matsubara Green's function $G^{M}(\tau), A(\epsilon)$ can be extracted by an analytic continuation to real energies and using the fluctuation-dissipation theorem (see Appendix B). Figures 1(b) and 1(c) show $A(\epsilon)$ for $\mu=0, \beta=5, v=-U / 2$, and four pairs $(U, \lambda)$. For $U=3$ and $\lambda=0.08$, the two main peaks correspond to the electronic excitation energies. Instead, for $\lambda=1$, phonon replicas spaced by $\omega$ are seen. $\mathrm{A}$ similar behavior occurs at $U=-1$ : for small $\lambda, A(\epsilon)$ has two main peaks. Here, the electron removal and addition parts of $A$ contribute to both peaks, since the $e$-ph interaction reorders the energy levels.

The zero-bias conductance $G$ is related to the spectral function [66]. Using $v_{x c}$ from Eq. (4), we calculate $G$ at zero temperature for a $\mathrm{HH}$ impurity connected to two 1D semiinfinite noninteracting leads. In this case, $G / G_{0}=\sin ^{2}(\pi n / 2)$
[67], with $n=(2 / \pi) \arctan \left(-v_{K S}[n] / \gamma \pi\right)+1, G_{0}$ being the unit of quantum conductance, and $\gamma$ being the level width in the wide-band limit. Results for $G$ as a function of $v$ and $\lambda$ are in Fig. 1(d), where $U=1$ and $\mu=0$. For $U^{\prime}>0$, $G$ has a plateau of width $U^{\prime}$ [56], but for $U^{\prime}<0$ we find a single narrow peak [23,67]. Overall, $G$ evolves smoothly as a function of $g^{2} / \omega$ between the spin- and charge-Kondo regimes.

Finally, we compare the exact double occupancy $\left\langle n_{\uparrow} n_{\downarrow}\right\rangle$ to the one obtained via DFT and the single-site potential. As shown in Appendix B, the exact double occupancy for the single-site system is given by

$$
\mathcal{D}^{s s}(\beta, n, U)=\left(\frac{1+\delta n}{2}\right) \frac{\delta n+R}{1+R} .
$$

In Fig. 1(e), we show $\left\langle n_{\uparrow} n_{\downarrow}\right\rangle$ for a seven-site chain with a $\mathrm{HH}$ impurity in the center. The exact $\mathcal{D}$ at the impurity is compared to $\mathcal{D}^{s s}$, which lacks kinetic energy effects but has the exact $n$ as input and thermal effects coming from a finite $\beta$. We see that for small $\beta$ the DFT result is in close agreement with the exact results, indicating that in some cases trading between kinetic and thermal effects can produce a nice agreement. However, the comparison also suggests that kinetic-energy effects in the screening by phonons are important, and a more general reference system than a single site should be used, as considered next.

\section{B. $v_{x c}$ from the infinite-dimensional Bethe lattice}

For the HH model on the $D=\infty$ Bethe lattice with bandwidth 4 (in units of the hopping parameter), we estimate the ground-state energy $E_{\text {tot }}$ within DMFT at $\beta=200$ [37]. The exchange-correlation potential is explicitly determined for $U=3$ and 8 and for $g=0.2$ and 1 , corresponding in all cases to a screened interaction $U^{\prime}>0$ [68]. Although not strictly at zero temperature, the value of $\beta$ is taken large enough that any finite-temperature effects should be negligible.

In Appendix C, we discuss in detail how the energy is extracted from the DMFT simulations. The results for the exchange correlation energy $E_{x c}$ are shown in Figs. 2(a)-2(d), and we find that the energy is smooth for small $U=3$ but has a kink at half-filling for $U=8$. This can be related to the presence of a Mott-Hubbard transition, as discussed further below. To perform the derivatives necessary in order to extract the exchange-correlation potential, $v_{x c}=\partial E_{x c}(n, x) / \partial n$, we fit the DMFT data in $n \in[0,1]$ with piecewise fourthorder polynomials. For $n \in[1,2]$, we employ the symmetry $E_{x c}(n)=E_{x c}(2-n)$.

In Figs. 2(e)-2(h), we show $v_{x c}$ obtained from the DMFT data. For $U=8, v_{x c}$ is discontinuous at $n=1$, while for $U=3$ the potential is smooth. This is a DFT signature of the Mott-Hubbard transition in the HH model, in analogy with the purely electronic Hubbard model [65] (for the $D=\infty$ Bethe lattice, when $\beta \rightarrow \infty, U_{c 1} \approx 4.7$, and $U_{c 2} \approx 5.8$ [69]). Interestingly, $e$-ph interactions not only renormalize the value of the XC discontinuity, but also delay its onset. For further insight, in Fig. 2 we also plot the single-site results from Eq. (4), with the value of $\beta$ fitted to best reproduce the DMFT curves (to see to what extent thermal fluctuations can mimic quantum fluctuations). For Figs. 2(e) and 2(f), this gives $\beta=5$ 

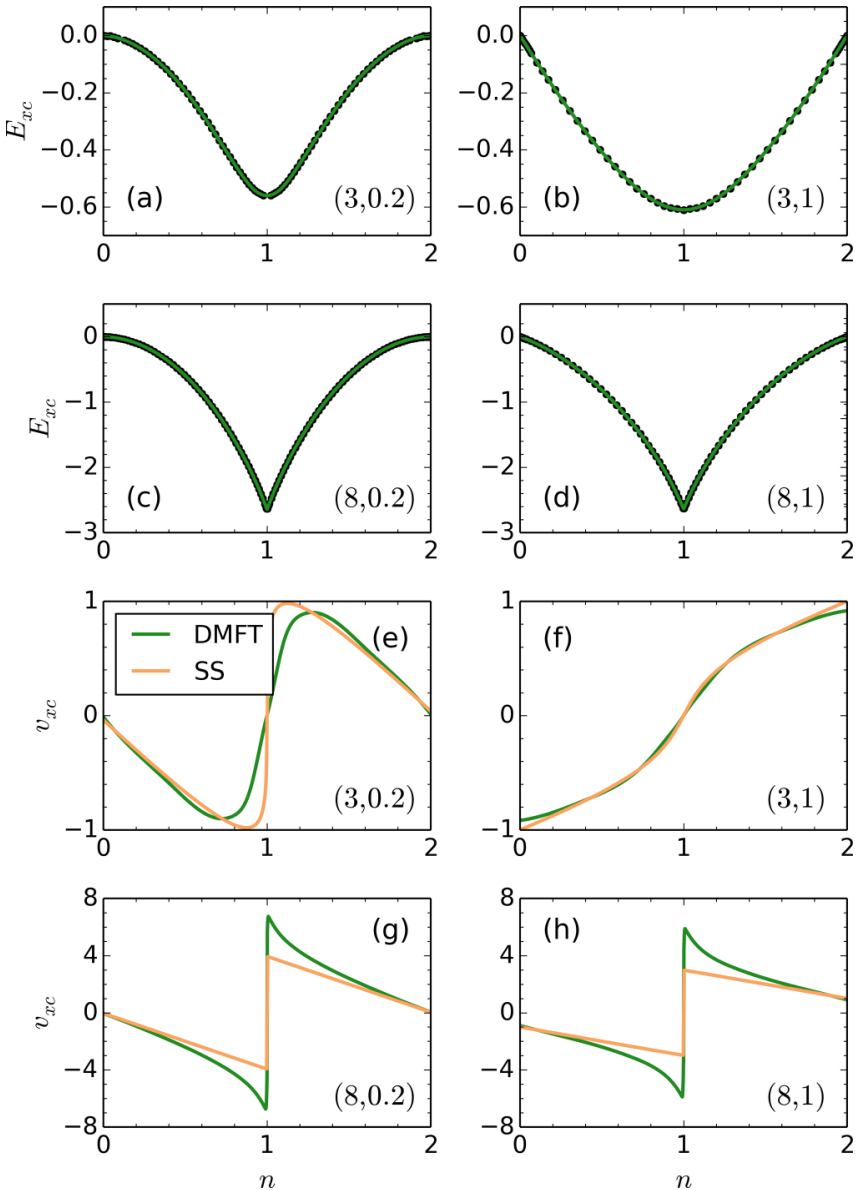

FIG. 2. Exchange-correlation energy $E_{x c}$ and exchangecorrelation potential $v_{x c}$ as a function of electron density $n, e-e$ interaction $U$, and $e$-ph interaction $g$. In all panels, the values of $(U, g)$ are shown, and $\omega=1$ and $\eta=0$. For the $E_{x c}$ results, the black dots are the DMFT data at $\beta=200$ and the green curves the numerical polynomial fit. For $v_{x c}$, the green curves show the polynomial fit of the DMFT data, and the yellow curves show the single Hubbard-Holstein site (SS) results. For the single-site potentials, $\beta$ is chosen via a fit to the DMFT results, giving $\beta \approx 5$ for $U=3$ and $\beta \approx 100$ for $U=8$.

and a smeared XC discontinuity. Also, due to the small $U$ and large $g$ values, the shape of the single-site solution in Fig. 2(f) is dominated by the linear term $g^{2} \delta n / \omega$. In contrast, in Figs. 2(g) and 2(h), the fit gives $\beta=100$ for the single-site potential, already close to the zero-temperature limit (where the discontinuity exists for all nonzero interactions $U^{\prime}$ for a zero-dimensional system). Overall, the single-site and DMFT potentials agree for $n \in[0,1 / 2]$ but significant differences appear at higher fillings, with important consequences for time-dependent simulations.

\section{REAL-TIME DYNAMICS}

We now use the single-site and DMFT potentials to investigate the real-time dynamics of an $L$-site chain with a $\mathrm{HH}$ impurity at one end (a so-called Anderson-Holstein chain; see Fig. 3, left). We are interested in the effects of dynamical phonon screening, present in the DMFT but not in the single-site potential. This system is chosen because the local density of states of a homogeneous Bethe lattice of coordination $Z$ and hopping amplitude $J$ is identical to the one at the first site of a semi-infinite chain. The mapping is obtained via Lanczos recursion and also holds with $\mathrm{HH}$ interactions and time-dependent fields at the central site of the Bethe lattice: The local Green's function at that site is the same as the one at site 0 of the chain. When $Z \rightarrow \infty$, the chain Hamiltonian is

$$
\begin{aligned}
H_{\text {chain }}= & -J \sum_{i=0, \sigma}^{\infty} c_{i \sigma}^{\dagger} c_{i+1, \sigma}+\text { H.c. } \\
& +v(t) \hat{n}_{0}+U \hat{n}_{0 \uparrow} \hat{n}_{0 \downarrow}+\omega b^{\dagger} b+g \hat{n}_{0}\left(b^{\dagger}+b\right),
\end{aligned}
$$

where $t$ labels time and $v(t)$ is a local perturbation.

In the simulations, we use $L=8$, which allows for exact numerical solutions. By virtue of the mapping, we are actually dealing with a $Z=\infty$ Bethe lattice truncated after eight layers and with one $\mathrm{HH}$ impurity in the center (Fig. 3). We consider $N_{\uparrow}=N_{\downarrow}=3$ electrons in the chain, and as before, $J=\omega=1$. The system's time evolution is obtained via exact diagonalization, as well as by TDDFT time propagation via the KS equations [70] within the adiabatic local density approximation (ALDA) [71]. By setting $v_{x c}$ to zero, we also consider the Hartree-Fock (HF) dynamics. Here we consider the electronic dynamics; the phonon results are instead shown in Appendix $\mathrm{D}$, since they essentially convey the same information as for the electronic case.

Figures 3(a)-3(c) show the dynamics after a sudden interaction quench $\left(U_{i}, g_{i}\right) \rightarrow\left(U_{f}, g_{f}\right)$ at $t=0$. This situation is within the scope of TDDFT, by freedom of choice of the initial state [27]. Further, quenches severely test the ALDA (typically employed within TDDFT). For the quench $(0,0) \rightarrow(3,1)$, Fig. 3(a), the exact and TDDFT-DMFT results are in excellent agreement, while the single-site and HF solutions give a moderately good description. Instead, for $(8,1) \rightarrow(3,1)$ and $(3,1) \rightarrow(8,1)$, Figs. 3(b) and 3(c), the agreement worsens, due to stronger interactions. However, the DMFT potential still qualitatively performs well, while the HF solution fails to capture the main features.

Figures 3(d)-3(f) show the dynamics induced by an external field $v(t)$, illustrated below each panel. In Fig. 3(d), where $U=8$ and $g=0.2, v(t)$ is a smooth step function ramped on in a time $\tau=8$ and is kept constant $(=1)$ afterward. There is excellent agreement between the exact and TDDFT results. In Figs. 3(e) and 3(f), the potential $v(t)$ is a soft square pulse of duration $\tau_{2}=4$ and amplitude $v=1$, switched on and off in a time $\tau_{1}=8$. In Fig. 3(e), where $U=8$ and $g=1$, the DMFT potential initially gives a very good agreement but this worsens near $n=1$. This is a known behavior and is due to the discontinuity of $v_{x c}$ at $n=1[59,65]$. Finally, we briefly turn to a case of $e$-ph overscreening (the $U^{\prime}<0$ region), using the single-site potential (the case of the DMFT potential is left for future work). The results of Fig. 3(f), where $U=-1$ and $g=0.5$, suggest that (TD)DFT can also be used for the attractive regime, but better potentials than the single-site one are clearly needed. Overall, the very good agreement between the exact and TDDFT-DMFT results indicates that dynamical phonon screening effects are an important ingredient of the 

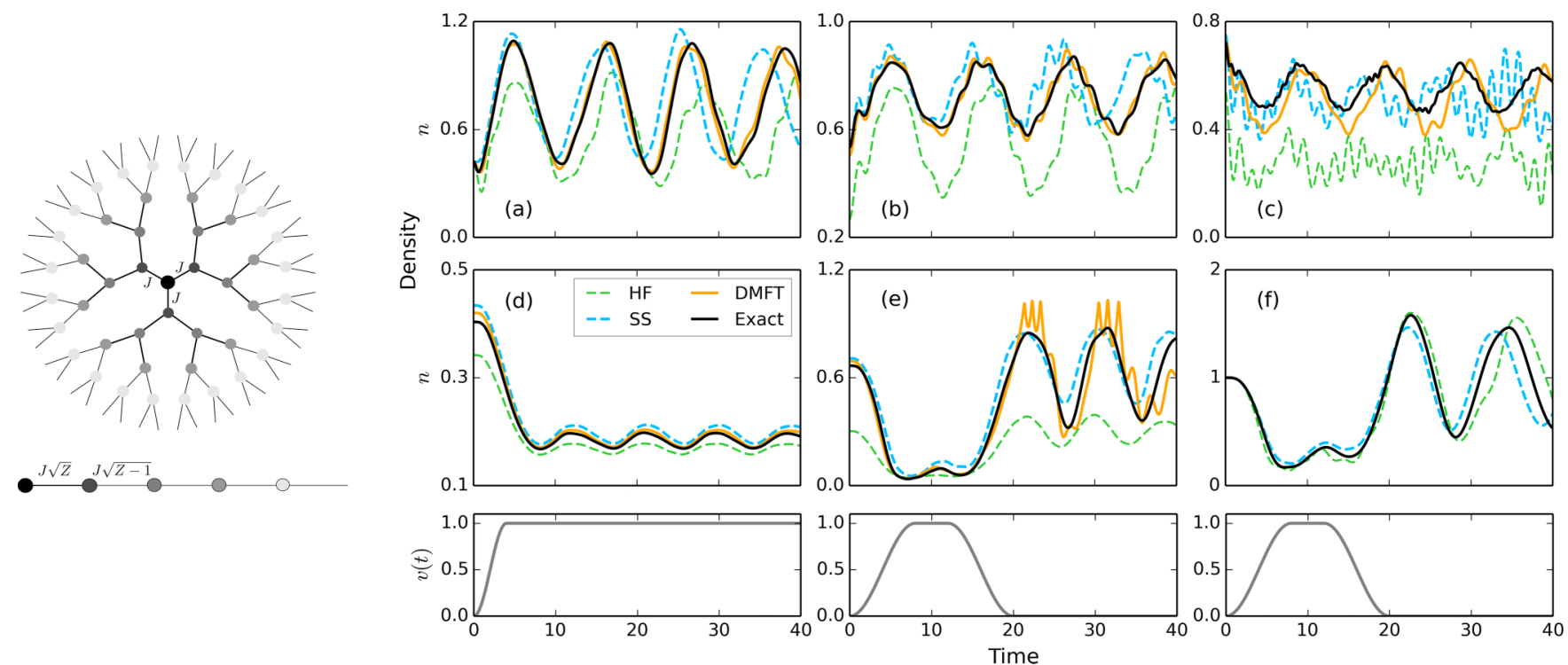

FIG. 3. Left: Mapping of the Bethe lattice with coordination number $Z$ onto a one-dimensional linear chain. For finite $Z$, the effective hoppings in the chain are $J \sqrt{Z}$ between sites 0 and 1 , and $J \sqrt{Z-1}$ otherwise. The results shown are for $Z \rightarrow \infty$ and renormalized hopping $J \rightarrow J / \sqrt{Z}$. Right: Dynamics of an eight-site Anderson-Holstein chain (see main text) with $n_{\downarrow}=n_{\uparrow}=3$, for $J=1, \omega=1$, and $Z \rightarrow \infty$. In all panels, the optimization of $\beta$ gives a value $\beta \simeq 5$. Panels (a)-(c) correspond to a sudden quench of the interaction pair $(U, g)$; from left to right, $(0,0) \rightarrow(3,1),(8,1) \rightarrow(3,1)$, and $(3,1) \rightarrow(8,1)$, respectively. In panel $(\mathrm{d}), U=8$ and $g=0.2$ and the external potential $v(t)$ is ramped to $v=1$ in a time $\tau=8$. For panels (e) and (f), the interactions are $U=8$ and $g=1$ or $U=-1$ and $g=0.5$, respectively, and the external field is a pulse of strength $v=1$ ramped on in a time $\tau_{1}=8$ and of duration $\tau_{2}=4$. The explicit shape of $v(t)$ is shown in the bottom panels.

$\mathrm{XC}$ potential and that the method is suitable and viable for inhomogeneous systems.

\section{CONCLUSIONS}

We have introduced a two-component density functional theory of electron-phonon lattice models, with exchangecorrelation potentials including nonperturbative phonon screening of the $e-e$ interactions. Our approach provides a good description of the conductance and real-time dynamics. Although the potentials considered here are for dimensions $D=0$ and $D=\infty$, an extension to $D=3$ is straightforward (but numerically demanding), which would pave the way for studies of strong $e$-ph interaction effects in large-scale, inhomogeneous systems. Appealing options for applications would be the analysis of the phonon overscreening regime, how phononlike degrees of freedom affect the physics of cold atoms in optical lattices, and the real-time dynamics in quantum transport geometries. Concerning more formal extensions, an immediate possibility is adapting the approach to the linear response regime. Further ahead, a key development would be the introduction of memory and nonlocal effects in the exchange-correlation potentials, by exploiting connections to many-body approximations within Green's function schemes.

\section{ACKNOWLEDGMENTS}

We thank C.-O Almbladh and A. Privitera for discussions. E.V.B. and P.H. were supported by Crafoordska stiftelsen. P.W. acknowledges support from ERC Consolidator Grant
No. 724103 and SNSF Grant No. 200021-165539. C.V. was supported by the Swedish Research Council.

\section{APPENDIX A: HOHENBERG-KOHN THEOREM AND KOHN-SHAM SYSTEM FOR THE HUBBARD-HOLSTEIN MODEL}

We here establish the Hohenberg-Kohn theorem for electron-phonon systems, by proving the existence of a bijective map between the pairs of conjugate variables $(\mu, \eta)$ and $(n, x)$. The proof is based on the constrained variational method introduced by Levý [72] and closely follows the line of reasoning of Schönhammer et al. [64]. However, a proof following directly the logic of the original HohenbergKohn formulation is also possible [73]. We then construct a Kohn-Sham system of noninteracting electrons and phonons, which for $v$ - and $\eta$-representable systems follows from the variational property of the total energy.

We assume the Hamiltonian to be of the form

$$
H=H_{0}+\sum_{i} v_{i} \hat{n}_{i}+\sqrt{2} \sum_{j} \eta_{j} \hat{x}_{j},
$$

where $\mu$ is an external potential acting on the electrons and $\eta$ is an external phonon potential. In the case of the HubbardHolstein model, we have

$$
\begin{aligned}
H_{0}= & -\mu \sum_{i \sigma} \hat{n}_{i \sigma}+U \sum_{i} \hat{n}_{i \uparrow} \hat{n}_{i \downarrow}-J \sum_{\langle i j\rangle \sigma} c_{i \sigma}^{\dagger} c_{j \sigma} \\
& +\omega \sum_{i} b_{i}^{\dagger} b_{i}+\sqrt{2} g \sum_{i}\left(\hat{n}_{i}-1\right) \hat{x}_{i} .
\end{aligned}
$$


For any state $|\Phi\rangle$, we define the energy by $E=\langle\Phi|H| \Phi\rangle$, the local electron density by $n_{i}=\left\langle\Phi\left|\hat{n}_{i}\right| \Phi\right\rangle$, and the mean phonon coordinate by $x_{j}=\left\langle\Phi\left|\hat{x}_{j}\right| \Phi\right\rangle$. To simplify the notation, we will use $n=\left\{n_{i}\right\}$ and $x=\left\{x_{j}\right\}$ to denote the full set of electronic densities and phononic displacements, and similarly $v=\left\{v_{i}\right\}$ and $\eta=\left\{\eta_{j}\right\}$ to denote the set of external potentials. The theorem we want to prove is the following:

Theorem (Hohenberg-Kohn). For given $v$ and $\eta$, let $\left|\Psi_{0}\right\rangle$ be the ground state of the Hamiltonian in Eq. (A1) with energy $E_{0}$. Then, the total energy $E$ of the system is a functional of the local electron densities $n_{i}$ and phonon coordinates $x_{j}$ and satisfies the variational property $E(n, x) \geqslant E_{0}$, with equality if and only if the densities and coordinates are given by their ground-state values.

Proof. We define $M(n, x)$ as the set of wave functions $|\Phi(n, x)\rangle$ that give the specific set of eigenvalues $n$ and $x$. Further, we define $F(n, x)$ as the smallest value in the set $M$, so that

$$
F(n, x)=\min _{|\phi\rangle \in M}\left\langle\phi\left|H_{0}\right| \phi\right\rangle .
$$

The total energy of the system is then given by

$$
E(n, x)=F(n, x)+\sum_{i} v_{i} \hat{n}_{i}+\sqrt{2} \sum_{j} \eta_{j} x_{j} .
$$

If we pick a state $|\Phi\rangle \in M$ such that $H_{0}$ attains its minimal value $F(n, x)$, we find

$$
\begin{aligned}
E(n, x) & =\left\langle\Phi\left|H_{0}\right| \Phi\right\rangle+\sum_{i} v_{i} n_{i}+\sqrt{2} \sum_{j} \eta_{j} x_{j} \\
& =F(n, x)+\sum_{i} v_{i} n_{i}+\sqrt{2} \sum_{j} \eta_{j} x_{j} \geqslant E_{0},
\end{aligned}
$$

where $E_{0}$ is the ground-state energy of $H$. This follows directly from the variational principle stating that any wave function $|\Phi\rangle$ will give an energy that is larger than or equal to the ground-state energy of the system.

We now pick another wave function $|\Psi\rangle$, which produces the ground-state energy $E_{0}$. In other words, $|\Psi\rangle$ is a ground state of the system described by $H$. We denote by $n_{i}^{0}=$ $\left\langle\Psi\left|\hat{n}_{i}\right| \Psi\right\rangle$ and $x_{i}^{0}=\left\langle\Psi\left|\hat{x}_{i}\right| \Psi\right\rangle$ the ground-state values of the density and position. Then, we obtain

$$
\begin{aligned}
E_{0} & =\left\langle\Psi\left|H_{0}\right| \Psi\right\rangle+\sum_{i} v_{i} n_{i}^{0}+\sqrt{2} \sum_{j} \eta_{j} x_{j}^{0} \\
& \geqslant F\left(n^{0}, x^{0}\right)+\sum_{i} v_{i} n_{i}^{0}+\sqrt{2} \sum_{j} \eta_{j} x_{j}^{0}=E\left(n^{0}, x^{0}\right) .
\end{aligned}
$$

This follows from the fact that although $|\Psi\rangle \in M\left(n^{0}, x^{0}\right)$, it may not be the state for which $F\left(n^{0}, x^{0}\right)$ is attained. Hence, we have obtained two inequalities $E(n, x) \geqslant E_{0}$ and $E\left(n^{0}, x^{0}\right) \leqslant$ $E_{0}$, but since the first inequality holds for all $(n, x)$, this in particular implies

$$
E(n, x) \geqslant E\left(n^{0}, x^{0}\right)=E_{0} .
$$

With this, we have shown that the total energy $E(n, x)$ is a functional of $n$ and $x$ that, for given $v$ and $\eta$, is minimized for the correct ground-state values $n^{0}$ and $x^{0}$. Furthermore, for a nondegenerate ground state, the density $n^{0}$ and displacement $x^{0}$ are completely determined by minimizing the total energy $E$.

We now use the Hohenberg-Kohn theorem to derive a set of noninteracting, coupled equations for the electrons and phonons that give the same ground-state expectation values $n^{0}$ and $x^{0}$ as the coupled system. We decompose the total energy $E[n, x]$ according to

$$
\begin{aligned}
E[n, x]= & T_{e}^{0}[n]+T_{\mathrm{ph}}^{0}[x]+E_{\mathrm{ext}}^{e}[n]+E_{\mathrm{ext}}^{\mathrm{ph}}[x]+E_{H}^{e}[n] \\
& +E_{H}^{e-\mathrm{ph}}[n, x]+E_{x c}[n, x],
\end{aligned}
$$

in order to separate the interaction energy from the noninteracting contributions. Here, the first two terms $T_{e}^{0}$ and $T_{\mathrm{ph}}^{0}$ give the kinetic energy of the noninteracting electron and phonon system, respectively, the next two terms $E_{\mathrm{ext}}^{e}$ and $E_{\mathrm{ext}}^{\mathrm{ph}}$ are the energies from the external potentials, followed by the Hartree energies $E_{H}^{e}$ and $E_{H}^{e-p h}$ of the electron-electron and electronphonon interaction. The last term is the exchange-correlation (xc) energy, containing everything left out by the other terms.

Since the total energy satisfies the variational principle with respect to $n$ and $x$ and the ground-state energy is a global minimum, the functional derivatives with respect to the variables $n$ and $x$ are both zero in the ground state. Taking the variation of the total energy yields

$$
\begin{aligned}
\delta E= & \sum_{i} \frac{\delta E[n, x]}{\delta n_{i}} \delta n_{i}+\sum_{j} \frac{\delta E[n, x]}{\delta x_{j}} \delta x_{j} \\
= & \sum_{i} \delta n_{i}\left(\frac{\delta T_{e}^{0}[n]}{\delta n_{i}}+v_{i}^{\mathrm{ext}}[n]+v_{i}^{H x c}[n, x]\right) \\
& +\sum_{j} \delta x_{j}\left(\frac{\delta T_{\mathrm{ph}}^{0}[x]}{\delta x_{j}}+\sqrt{2} \eta_{j}^{\mathrm{ext}}[x]+\sqrt{2} \eta_{j}^{H x c}[n, x]\right),
\end{aligned}
$$

where in the last line we have introduced the Hartree exchange-correlation potentials $v^{H x c}=v_{e}^{H}+v_{e-\mathrm{ph}}^{H}+v^{x c}$ and $\eta^{H x c}=\eta_{e-\mathrm{ph}}^{H}+\eta^{x c}$ for the electron and phonon systems. Demanding that $\delta E=0$ for arbitrary variations $\delta n$ and $\delta x$, this gives the equations of motion of the system.

We now recognize that the above expression can also be obtained by taking the variation of the energy corresponding to the Hamiltonian

$$
\begin{aligned}
H_{K S}= & -\mu \sum_{i \sigma} \hat{n}_{i \sigma}-J \sum_{\langle i j\rangle \sigma} c_{i \sigma}^{\dagger} c_{j \sigma}+\sum_{i \sigma} v_{i}^{K S}[n, x] \hat{n}_{i \sigma} \\
& +\omega \sum_{j} b_{j}^{\dagger} b_{j}+\sqrt{2} \sum_{j} \eta_{j}^{K S}[n, x] \hat{x}_{j} \\
= & H_{0}+\sum_{i \sigma} v_{i}^{K S}[n, x] \hat{n}_{i \sigma}+\sqrt{2} \sum_{j} \eta_{j}^{K S}[n, x] \hat{x}_{j},
\end{aligned}
$$

where the Kohn-Sham potentials are $v_{i}^{K S}=v_{i}^{\text {ext }}+v_{i}^{H x c}$ and $\eta_{j}^{K S}=\eta_{j}^{\text {ext }}+\eta_{j}^{H x c}$. These equations need to be solved with the additional restriction that the ground state of $H_{K S}$ reproduces the correct ground-state electron density and phonon displacement. This leads to the coupled pair of Kohn-Sham equations $H_{K S}^{e}\left|\psi_{m}\right\rangle=\varepsilon_{m}^{e}\left|\psi_{m}\right\rangle$ and $H_{K S}^{\mathrm{ph}}\left|\chi_{n}\right\rangle=\varepsilon_{n}^{\mathrm{ph}}\left|\chi_{n}\right\rangle$ where 
the electron and phonon Hamiltonians are respectively given by

$$
\begin{aligned}
& H_{K S}^{e}=-J \sum_{\langle i j\rangle \sigma}\left(c_{i \sigma}^{\dagger} c_{j \sigma}+\text { H.c. }\right)+\sum_{i \sigma} v_{i}^{K S}[n, x] \hat{n}_{i \sigma}, \\
& H_{K S}^{\mathrm{ph}}=\sum_{j}\left(\omega b_{j}^{\dagger} b_{j}+\sqrt{2} \eta_{j}^{K S}[n, x] \hat{x}_{j}\right) .
\end{aligned}
$$

The Hohenberg-Kohn theorem and Kohn-Sham equations provide a firm basis for the application of ground-state DFT to the $\mathrm{HH}$ model. To justify the use of a time-dependent treatment, a proof similar to that originally given for electronphoton lattice systems [33] (with electron densities and photon momenta as fundamental variables) should be adapted to the present case. However, since this adaptation is straightforward, it is not discussed in detail here.

The time-dependent Kohn-Sham equations corresponding to Eqs. (A11) above, are given by

$$
\begin{aligned}
i \partial_{t}\left|\psi_{m}\right\rangle & =\hat{H}^{e}(t)\left|\psi_{m}\right\rangle \\
& =\left[-J \sum_{\langle i j\rangle \sigma}\left(\hat{c}_{i \sigma}^{\dagger} \hat{c}_{j \sigma}+h . c\right)+\sum_{i \sigma} v_{i}^{K S}[n, x](t) \hat{n}_{i \sigma}\right]\left|\psi_{m}\right\rangle, \\
i \partial_{t}\left|\chi_{n}\right\rangle & =\hat{H}^{\mathrm{ph}}(t)\left|\chi_{n}\right\rangle=\sum_{j}\left[\omega \hat{b}_{j}^{\dagger} \hat{b}_{j}+\sqrt{2} \eta_{K S}[n, x](t) \hat{x}_{j}\right]\left|\chi_{n}\right\rangle .
\end{aligned}
$$

These equations are used to study the dynamics of the Hubbard-Holstein system in Fig. 3. To solve the Kohn-Sham equations, we use the adiabatic local density approximation, which means we assume $v_{i}^{K S}[n, x](t) \approx v_{i}^{K S}\left(n_{i}(t), x_{i}(t)\right)$ and $\eta_{j}^{K S}[n, x](t) \approx \eta_{j}^{K S}\left(n_{j}(t), x_{j}(t)\right)$. If we further take the external phonon potential to vanish, the phononic Kohn-Sham potential is $\eta_{j}^{K S}[n, x](t) \approx g\left(n_{j}(t)-1\right)$.

\section{APPENDIX B: RESULTS FOR A SINGLE HUBBARD-HOLSTEIN SITE}

In this section, we discuss some properties of a single Hubbard-Holstein site, where many quantities can be obtained analytically. We first calculate the exchange-correlation potential for the electrons, then obtain the thermal spectral function of the system, and lastly find an expression for the double occupancy in terms of the electron density.

\section{Exchange-correlation potential}

We here find the exact exchange-correlation potential of a single Hubbard-Holstein site. After a Lang-Firsov transformation, the single-site Hamiltonian is given by

$$
H^{\prime}=\left(v^{\prime}-\mu\right) \hat{n}+U^{\prime} \hat{n}_{\uparrow} \hat{n}_{\downarrow}+\omega b^{\dagger} b-E_{p},
$$

with the renormalized potential $v^{\prime}=v-g^{2} / \omega-2 g \eta / \omega+$ $2 g^{2} n_{0} / \omega$, the screened interaction $U^{\prime}=U-2 g^{2} / \omega$, and the polaron energy $E_{p}=\left(\eta-g n_{0}\right)^{2} / \omega$.

To calculate the electronic exchange-correlation potential, we start by evaluating the partition function $Z=\operatorname{Tr}\left(e^{-\beta H^{\prime}}\right)$.
This is given by

$$
Z=\frac{e^{\beta E_{p}}}{1-e^{-\beta \omega}}\left[1+2 e^{\beta\left(\mu-v^{\prime}\right)}+e^{\beta\left(2 \mu-U-2 v^{\prime}\right)}\right]
$$

and can be used to calculate the density from $n=(-\beta)^{-1} \partial_{v}$ $\ln Z$. Doing so, we obtain the expression

$$
n=2 \frac{e^{\beta\left(\mu-v^{\prime}\right)}+e^{\beta\left(2 \mu-U-2 v^{\prime}\right)}}{1+2 e^{\beta\left(\mu-v^{\prime}\right)}+e^{\beta\left(2 \mu-U-2 v^{\prime}\right)}} .
$$

Inverting this relation to express $v^{\prime}-\mu$ in terms of $n$ and afterward solving for the original potential $v$ making use of the expression $\eta=-\omega x / \sqrt{2}-g\left(n-n_{0}\right)$ to eliminate $\eta$, we find

$$
\begin{aligned}
v-\mu= & -U^{\prime}-\frac{1}{\beta} \ln \left[\frac{\delta n+\sqrt{\delta n^{2}+e^{-\beta U^{\prime}\left(1-\delta n^{2}\right)}}}{1-\delta n}\right] \\
& +\frac{g^{2}}{\omega}-\frac{2 g^{2}}{\omega} n-\sqrt{2} g x .
\end{aligned}
$$

To finally obtain the exchange-correlation potential, we note that the Kohn-Sham potential $v_{k s}$, which by definition reproduces the correct density and phonon coordinate in a noninteracting system, is obtained by evaluating the expression above at $U=g=0$. Since $v_{x c}=v_{k s}-v-v_{H}$, where $v_{H}$ is the Hartree potential, we find

$$
\begin{aligned}
v_{x c}= & \frac{U^{\prime}}{2}(1-\delta n)+\frac{g^{2}}{\omega} \delta n \\
& +\frac{1}{\beta} \ln \left[\frac{\delta n+\sqrt{\delta n^{2}+e^{-\beta U^{\prime}}\left(1-\delta n^{2}\right)}}{1+\delta n}\right] .
\end{aligned}
$$

This is the expression for $v_{x c}$ presented in the main text. We note that it reduces to the known expression for $v_{x c}$ for a single Hubbard site when $g=0$, and also that the final expression is independent of $n_{0}$. This means it applies equally well both to the particle-hole symmetric case and the nonsymmetric case.

\section{Spectral function}

The thermal spectral function $A(\epsilon)$ can be calculated by evaluating the Matsubara Green's function $G^{M}(\tau)=$ $(i Z)^{-1} \operatorname{Tr}\left(e^{-\beta H} \mathcal{T}\left[c(\tau) c^{\dagger}(0)\right]\right)$, and later making a Fourier transforming to energy. Since in the Fourier transform of $G^{M}$ we can restrict to $\tau>0$, we need only evaluate this part of $G^{M}$. For a single Hubbard-Holstein site, one obtains

$$
\begin{aligned}
G^{M}(\tau)= & \frac{1}{i Z} \sum_{\alpha \sigma m}\left\langle\alpha m\left|e^{-\beta H^{M}} c_{\sigma}(\tau) c_{\sigma}^{\dagger}(0)\right| \alpha m\right\rangle \\
= & \frac{2}{i Z} \sum_{m}\left[e^{\tau(\mu-v)}\left\langle m\left|e^{(\tau-\beta)\left(\omega b^{\dagger} b-g\left(b^{\dagger}+b\right)\right)} e^{-\tau \omega b^{\dagger} b}\right| m\right\rangle\right. \\
& \left.+e^{\beta(\mu-v)} e^{\tau(\mu-U-v)}\left\langle m\left|e^{(\tau-\beta) \omega b^{\dagger} b} e^{-\tau\left(\omega b^{\dagger} b+g\left(b^{\dagger}+b\right)\right)}\right| m\right\rangle\right],
\end{aligned}
$$

where $\alpha$ is a sum over the electronic states and the Hamiltonian on the Matsubara branch is $H^{M}=H-\mu N$. To calculate this expression, we need to evaluate matrix elements of the general form

$$
M=\left\langle m\left|e^{(\tau-\beta)\left(\omega b^{\dagger} b+f\left(b^{\dagger}+b\right)\right)} e^{-\tau\left(\omega b^{\dagger} b+f^{\prime}\left(b^{\dagger}+b\right)\right)}\right| m\right\rangle .
$$


This is efficiently done by noting that, since we trace over a complete set of phonon states, we can choose these states as eigenstates of a shifted harmonic oscillator that satisfy $\left[\omega b^{\dagger} b+f\left(b^{\dagger}+b\right)\right]|m, f\rangle=\left(\omega m-f^{2} / \omega\right)|m, f\rangle$. These states are related to the unshifted harmonic oscillator states by $|m, f\rangle=\Lambda(f / \omega)|m\rangle$, with the translation operator $\Lambda(f / \omega)=e^{f / \omega\left(b^{\dagger}-b\right)}$ (this follows from a simplified version of the Lang-Firsov transformation). The matrix element $M$ can now be written as

$$
M=e^{(\tau-\beta)\left(\omega m-f^{2} / \omega\right)} \sum_{k} e^{-\tau\left(\omega k-f^{\prime 2} / \omega\right)}\left|\left\langle k, f^{\prime} \mid m, f\right\rangle\right|^{2},
$$

where we have inserted a complete set of states $\left|k, f^{\prime}\right\rangle$. What remains is the calculation of the overlap between shifted harmonic oscillator states. This was done, e.g., by Cahill and Glauber [75], who found they can be written in terms of the associated Laguerre polynomials $L_{n}^{(\alpha)}$. For the case considered here, we always have $f+f^{\prime}= \pm g$, the overlaps can be expressed compactly as $\left|\left\langle k, f^{\prime} \mid m, f\right\rangle\right|^{2}=e^{-g^{2} / \omega^{2}} F_{m k}\left(g^{2} / \omega^{2}\right)$, where the function $F_{m k}$ is given by

$$
\begin{aligned}
& F_{m k}(x)=\frac{k !}{m !} x^{(m-k)}\left[L_{m}^{(k-m)}(x)\right]^{2} \quad \text { if } \quad k \leqslant m, \\
& F_{m k}(x)=\frac{m !}{k !} x^{(k-m)}\left[L_{k}^{(m-k)}(x)\right]^{2} \quad \text { if } \quad k \geqslant m .
\end{aligned}
$$

This gives the final expression for the $\tau>0$ part of $G^{M}$ as

$$
\begin{aligned}
G^{M}(\tau)= & 2 \sum_{m k}\left[e^{-\beta\left(\omega m-g^{2} / \omega\right)} e^{-\tau\left(\omega(k-m)+v-\mu-g^{2} / \omega\right)}\right. \\
& \left.+e^{-\beta(\omega m+v-\mu)} e^{-\tau\left(\omega(k-m)+U+v-\mu+g^{2} / \omega\right)}\right] e^{-g^{2} / \omega^{2}} \\
& \times F_{m k}\left(\frac{g^{2}}{\omega^{2}}\right) .
\end{aligned}
$$

Having evaluated $G^{M}(\tau)$, we can transform to energy using $G^{M}\left(i \omega_{n}\right)=\int d t e^{i \omega_{n} \tau} G^{M}(\tau)$, where $\omega_{n}=(2 n+1) \pi / \beta$. To get the lesser and greater components of the spectral function, we first perform the analytic continuation to real energies, $G^{R / A}(\epsilon)=G^{M}(\epsilon-\mu \pm i \delta)$, where $\delta$ is a positive infinitesimal, and then use the fluctuation-dissipation theorem $G^{<}(\epsilon)=-f(\epsilon-\mu)\left[G^{R}(\epsilon)-G^{A}(\epsilon)\right]$ and $G^{>}(\epsilon)=$ $\bar{f}(\epsilon-\mu)\left[G^{R}(\epsilon)-G^{A}(\epsilon)\right]$, where $f$ is the Fermi-Dirac distribution and $\bar{f}=1-f$. Putting these expressions together, we find the greater and lesser parts of the spectral function to be

$$
\begin{aligned}
A^{>}(\epsilon)= & \frac{e^{-g^{2} / \omega^{2}}}{Z} \sum_{m k} F_{m k}\left(\frac{g^{2}}{\omega^{2}}\right)\left[e^{-\beta\left(\omega m-g^{2} / \omega\right)} \delta(\epsilon-\omega(k-m)\right. \\
& \left.-v+g^{2} / \omega\right)+e^{-\beta(\omega m+v-\mu)} \delta(\epsilon-\omega(k-m) \\
& \left.\left.-U-v-g^{2} / \omega\right)\right], \\
A^{<}(\epsilon)= & \frac{e^{-g^{2} / \omega^{2}}}{Z} \sum_{m k} F_{m k}\left(\frac{g^{2}}{\omega^{2}}\right)\left[e^{-\beta(\omega k+v-\mu)} \delta(\epsilon-\omega(k-m)\right. \\
& \left.-v+g^{2} / \omega\right)+e^{-\beta\left(\omega k+U+2 v-2 \mu-g^{2} / \omega\right)} \\
& \left.\times \delta\left(\epsilon-\omega(k-m)-U-v-g^{2} / \omega\right)\right],
\end{aligned}
$$

where the partition function is given by $Z=e^{\beta g^{2} / \omega}$ $\left(1-e^{-\beta \omega}\right)^{-1}\left[1+2 e^{\beta(\mu-v)}+e^{\beta(2 \mu-U-2 v)}\right]$. To obtain the expression for the greater part, we have used that the function $\bar{f}$ cancels the numerator coming from the integration over $\tau$, when evaluated at the energy given by the $\delta$ function. Similarly, we have simplified the expression for the lesser part using a cancellation of the Fermi function. These expressions have the general structure of a sum of peaks at the addition (removal) energies of the system, weighted by the thermal occupation of the initial state and an overlap of quasiparticle amplitudes.

As a consistency check, we note that in the limit $\beta \rightarrow \infty$, this formula should reproduce the zero-temperature expression obtained by Langreth [76]. To obtain his expression, we need to start from the state $|\uparrow, 0\rangle$ with one electron and zero phonons, instead of taking the trace over all states. This amounts to taking $m=0$ in the expression above, and neglecting the two last terms that come from the initial state $|\uparrow \downarrow, 0\rangle$. In this limit, the partition function becomes $Z=e^{\beta g^{2} / \omega} /\left(1-e^{-\beta \omega}\right)$, and we find

$$
\begin{aligned}
A^{>}(\epsilon) & =\frac{e^{-g^{2} / \omega^{2}+\beta g^{2} / \omega}}{Z} \sum_{k} F_{0 k}\left(\frac{g^{2}}{\omega^{2}}\right) \delta\left(\epsilon-\omega k-v+\frac{g^{2}}{\omega}\right) \\
& =e^{-g^{2} / \omega^{2}} \sum_{k} \frac{1}{k !}\left(\frac{g^{2}}{\omega^{2}}\right)^{k} \delta\left(\epsilon-\omega k-v+\frac{g^{2}}{\omega}\right),(\mathrm{B} 11)
\end{aligned}
$$

where we have used that $F_{0 k}(x)=x^{k} / k$ !. As expected, this gives the known zero-temperature expression for $A^{<}$.

\section{Double occupancy}

To gain some insights into the interplay of $e$ - $e$ and $e$ $\mathrm{ph}$ interactions, it is useful to look at the double occupancy $\mathcal{D} \equiv\left\langle n_{\uparrow} n_{\downarrow}\right\rangle=-\frac{1}{\beta} \partial_{U} \ln Z$. For the single-site system, this quantity can be obtained as an explicit function of the electron density $n$. This is done by noting that $\mathcal{D} / n=$ $\left[2+2 e^{\beta(U+v-\mu)}\right]^{-1}$, and by using the expression for $v$ given in the main text, we have $e^{\beta(U+v-\mu)}=(1-\delta n) /(\delta n+R)$ with $R=\sqrt{\delta n^{2}+e^{-\beta U}\left(1-\delta n^{2}\right)}$. The exact DFT expression for the single-site system is therefore

$$
\mathcal{D}^{s s}(\beta, n, U)=\left(\frac{1+\delta n}{2}\right) \frac{\delta n+R}{1+R} .
$$

\section{APPENDIX C: TOTAL ENERGY OF THE HOMOGENEOUS SYSTEM}

To find the exchange-correlation potential of the electronic system, we calculate the total energy of the homogeneous reference system obtained by taking $v_{i}=v$ and $\eta_{j}=\eta$. This implies that all local observables are independent of the site index, so that $n_{i}=n$ and $x_{i}=x$. We also note, as discussed in the main text, that the phonon exchange-correlation potential vanishes identically.

To simplify the numerical evaluation of the ground-state energy, the electron-phonon interaction can be shifted to the kinetic energy term using the Lang-Firsov transformation $H^{\prime}=e^{i S} H e^{-i S}$, where

$$
S=\frac{\sqrt{2}}{\omega} \sum_{i}\left[g\left(\hat{n}_{i}-1\right)+\eta\right] \hat{p}_{i},
$$


and $\hat{p}_{i}=i\left(b_{i}^{\dagger}-b_{i}\right) / \sqrt{2}$ is the phonon momentum. Using the Baker-Hausdorff formula to perform the manipulations, we find the transformed Hamiltonian

$$
\begin{aligned}
H^{\prime}= & \sum_{i \sigma}\left(v^{\prime}-\mu\right) \hat{n}_{i \sigma}+\sum_{i} U^{\prime} \hat{n}_{i \uparrow} \hat{n}_{i \downarrow}-\sum_{\langle i j\rangle \sigma} \hat{J}_{i j}^{\prime} c_{i \sigma}^{\dagger} c_{j \sigma} \\
& +\sum_{i}\left[\omega b_{i}^{\dagger} b_{i}-\frac{(\eta-g)^{2}}{\omega}\right]
\end{aligned}
$$

where the parameters are given by $v^{\prime}=v+\left(g^{2}-2 g \eta\right) / \omega$, $U^{\prime}=U-2 g^{2} / \omega$, and $\hat{J}_{i j}^{\prime}=J \exp \left[i \sqrt{2} g\left(\hat{p}_{i}-\hat{p}_{j}\right) / \omega\right]$. We note that the hopping amplitude is now an operator which acts on the phonon states.

To construct the exchange-correlation potentials, we need to evaluate the ground-state energy of the system for all $n$ and $x$. For given values of the interaction $U$, electron-phonon coupling $g$, and phonon frequency $\omega$, this in principle means that we need to solve the model for all values of $v, \mu$, and $\eta$. The calculation is, however, simplified by noting that the transformed Hamiltonian $H^{\prime}$ depends only on the effective parameter $-\mu^{\prime}=v^{\prime}-\mu$ (up to an $\eta$-dependent constant). In addition, for a homogeneous system, $v$ is a redundant variable that can be set to zero by a suitable redefinition of the chemical potential. Although $\mu^{\prime}$ still carries an implicit dependence on $\eta$, in practice we can treat $\mu^{\prime}$ as an independent parameter.

To determine the ground-state energy $E_{0}[\mu, \eta]$, it suffices to find its value for all $\mu^{\prime}$ and $\eta=0$. The energy for $\eta \neq 0$ can then be obtained from the case where $\eta=0$ through the relation

$$
E_{0}[\mu, \eta]=E_{0}\left[\mu^{\prime}(\mu, \eta)\right]-\frac{(\eta-g)^{2}}{\omega} .
$$

Since in the end we want the energy as a function of the density $n$ and position $x$, we need to relate their values for general $\mu$ and $\eta$ to the case where $\eta=0$. However, it follows from the Heisenberg equation of motion for the phonon momentum, $\partial_{t} p=i \hbar^{-1}[H, p]=0$, that $x=-\sqrt{2}[g(n-1)+\eta] / \omega$. This means that the phonon coordinate $x$ is completely determined by $n$ and $\eta$. To obtain the energy as a function of $n$ and $x$, it is therefore sufficient to calculate $E_{0}$ and $n$ as a function of $\mu^{\prime}$ (for $\eta=0$ ), which allows us to determine $n$ as a function of $\mu^{\prime}$. We can then determine $E_{0}$ for all values of $\eta$ and finally invert the relation above to obtain $x$ as a function of $n$ and $\eta$. This completely determines $E_{0}[n, x]$, as shown in more detail below.

An explicit expression for the exchange-correlation energy is obtained by first writing the total energy as the sum of terms in Eq. (C2). We eliminate the variables $\mu^{\prime}$ and $\eta$ by writing them in terms of $n$ and $x$. Using that $\eta=-g(n-1)-$ $\omega x / \sqrt{2}$, we find that the term proportional to $\mu^{\prime}$ is given by

$$
-\mu^{\prime} n_{i}=-\mu n_{i}-\frac{g^{2} n_{i}}{\omega}+\sqrt{2} g n_{i} x_{i}+\frac{2 g^{2} n_{i}^{2}}{\omega},
$$

while the constant term can be expanded as

$$
-\frac{(\eta-g)^{2}}{\omega}=-\frac{\omega x_{i}^{2}}{2}-\frac{g^{2} n_{i}^{2}}{\omega}-\sqrt{2} g n_{i} x_{i} .
$$

For the total energy, we then find the expression

$$
\begin{aligned}
E=T[n]+\sum_{i} & {\left[U^{\prime}\left\langle n_{i \uparrow} n_{i \downarrow}\right\rangle+\omega n_{i, \mathrm{ph}}-\mu n_{i}\right.} \\
& \left.+\frac{g^{2} n_{i}}{\omega}\left(n_{i}-1\right)-\frac{\omega x_{i}^{2}}{2}\right],
\end{aligned}
$$

where $T[n]=-\sum_{\langle i j\rangle \sigma}\left\langle\hat{J}_{i j}^{\prime} c_{i \sigma}^{\dagger} c_{j \sigma}\right\rangle$ is the kinetic energy of the electrons. To obtain the exchange-correlation energy $E_{x c}$, we write it in the form

$$
E_{x c}=E-E_{\mathrm{ext}}^{e}-E_{\mathrm{ext}}^{\mathrm{ph}}-E_{H}^{e-e}-E_{H}^{e-\mathrm{ph}}-E_{0}^{e}-E_{0}^{\mathrm{ph}} .
$$

The external energies are given respectively by $E_{\mathrm{ext}}^{e}=$ $-\mu \sum_{i} n_{i}$ and $E_{\mathrm{ext}}^{\mathrm{ph}}=\sqrt{2} \eta \sum_{i} x_{i}=-\sum_{i}\left[\omega x_{i}^{2}+\sqrt{2} g x_{i}\left(n_{i}-1\right)\right]$, while the Hartree contributions from the electron-electron and electron-phonon interactions are $E_{H}^{e-e}=(U / 4) \sum_{i} n_{i}^{2}$ and $E_{H}^{e-\mathrm{ph}}=\sqrt{2} g \sum_{i} x_{i}\left(n_{i}-1\right)$. For the phonons, we find the noninteracting energy

$$
E_{0}^{\mathrm{ph}}=\sum_{i}\left[\omega n_{i, \mathrm{ph}}-\frac{\eta_{0}^{2}}{\omega}-\sqrt{2} \eta_{0} x_{i}\right]=\frac{\omega}{2} \sum_{i} x_{i}^{2},
$$

where we have used that for a noninteracting system $x_{i}=$ $-\sqrt{2} \eta_{0} / \omega$. Denoting the kinetic energy of the noninteracting electron system by $T_{0}[n]=-J \sum_{\langle i j\rangle \sigma}\left\langle c_{i \sigma}^{\dagger} c_{j \sigma}\right\rangle_{0}$ (taken over the noninteracting ground state), we obtain the exchangecorrelation energy

$$
\begin{aligned}
E_{x c}=T[n]-T_{0}[n]+\sum_{i} & {\left[U^{\prime}\left\langle n_{i \uparrow} n_{i \downarrow}\right\rangle+\omega n_{i, \mathrm{ph}}-U \frac{n_{i}^{2}}{4}\right.} \\
& \left.+\frac{g^{2} n_{i}}{\omega}\left(n_{i}-1\right)\right] .
\end{aligned}
$$

We used dynamical mean-field theory (DMFT) to calculate the total energy $E$ and constructed the exchange-correlation energy from the expression above. In practice, this was done by measuring the observables in Eq. (C9) with DMFT, namely the kinetic energy, the double occupancy, the electron density, and the phonon density. The hybridization expansion continuous-time Monte Carlo approach [74] allows us to obtain the kinetic energy from the average perturbation order, and the density and double occupation from the average length and overlap of the spin-up and spin-down segments. To measure the phonon occupation, we insert the operator $O=e^{x b^{\dagger}-y b}$ at imaginary time $\tau=0$ and calculate the corresponding Monte Carlo weights following Ref. [37]. Taking appropriate derivatives with respect to $x$ and $y$ and setting $x=y=0$ leads to the measurement formula for $n_{\mathrm{ph}}$.

\section{APPENDIX D: PHONON DYNAMICS}

In Fig. 4, we show the dynamics of the phonon coordinate $x$ at site 0 , corresponding to the electron density of Fig. 3 in the main text. The parameters and perturbation are exactly the same as those of Fig. 3.

In Figs. 4(a)-4(c) we study a sudden interaction quench $\left(U_{i}, g_{i}\right) \rightarrow\left(U_{f}, g_{f}\right)$ at $t=0$. For the quench $(0,0) \rightarrow(3,1)$ shown in Fig. 4(a), the exact and TDDFT-DMFT results are in excellent agreement, while the single-site potential and the HF solution give a moderately good description. Instead, for 

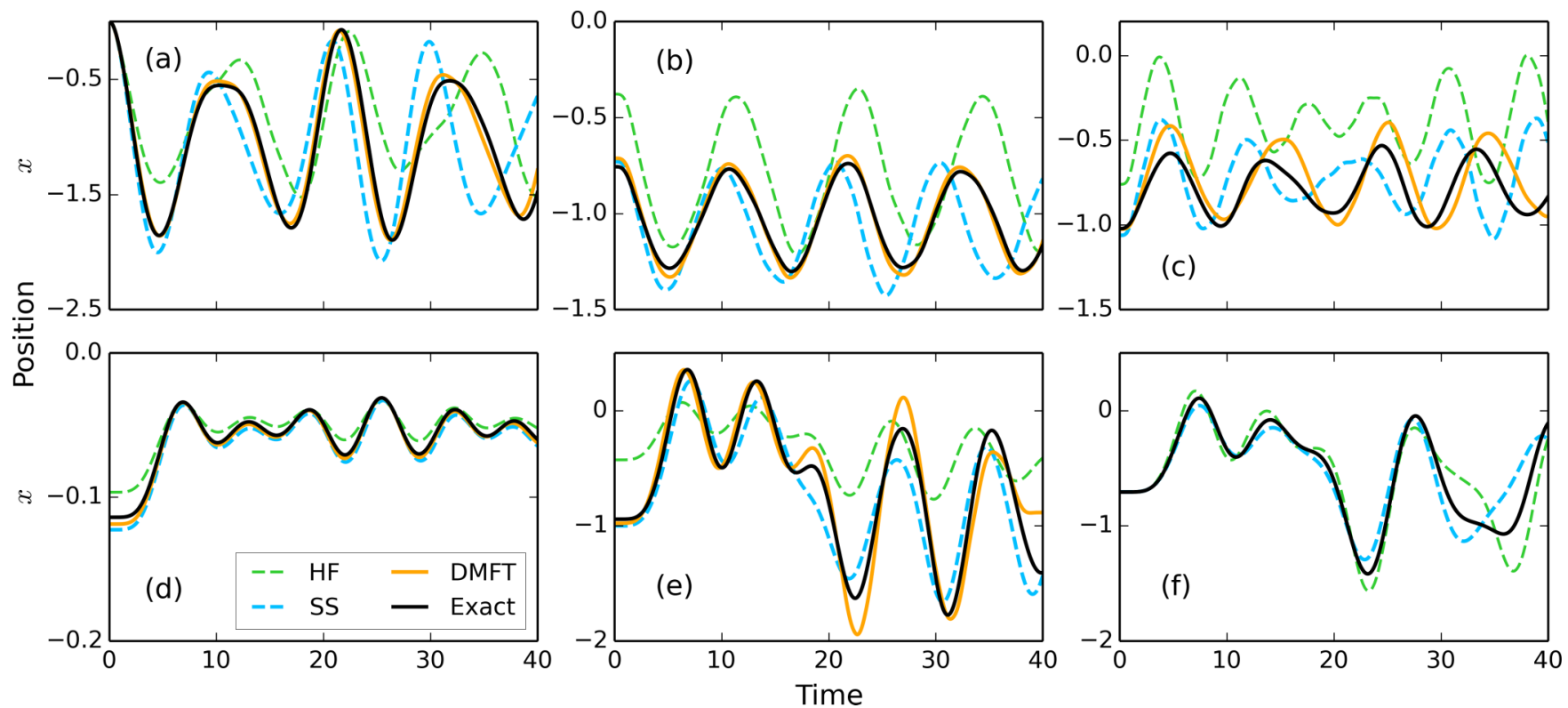

(c)

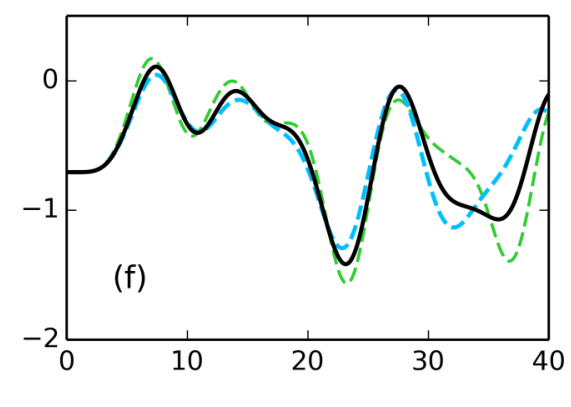

FIG. 4. Dynamics of an eight-site Anderson-Holstein chain with $n_{\downarrow}=n_{\uparrow}=3$, for $J=1, \omega=1$, and $Z \rightarrow \infty$. In all panels, the optimization of $\beta$ gives a value $\beta \simeq 5$. Panels (a)-(c) correspond to a sudden quench of the interaction pair $(U, g$ ); from left to right, $(0,0) \rightarrow(3,1),(8,1) \rightarrow(3,1)$, and $(3,1) \rightarrow(8,1)$, respectively. In panel $(\mathrm{d}), U=8$ and $g=0.2$ and the external potential is ramped to $v=1$ in a time $\tau=8$. For panels (e) and (f), the interactions are $U=8$ and $g=1$ or $U=-1$ and $g=0.5$ respectively, and the external field is a pulse of strength $v=1$ ramped on in a time $\tau_{1}=8$ and of duration $\tau_{2}=4$.

$(8,1) \rightarrow(3,1)$ and $(3,1) \rightarrow(8,1)$ as shown in Figs. $4(b)$ and $4(\mathrm{c})$, the agreement worsens due to stronger interactions. However, the DMFT potential still qualitatively performs well, while the HF solution fails to capture the main features.

Figures 4(d)-4(f) show the dynamics induced by an external field $v(t)$ applied at the impurity site. In Fig. 4(d), where $U=8$ and $g=0.2, v(t)$ is ramped on in a time $\tau=8$, and kept constant afterward. In Figs. 4(e) and 4(f), the potential $v(t)$ is a smoothed square pulse of duration $\tau_{2}=4$, ramped on and off in a time $\tau_{1}=8$. In Fig. 4(e), where $U=8$ and $g=1$, the DMFT potential gives a good agreement, while the single-site potential is slightly worse. In both cases, there is no trace of the artificial oscillation seen in the electron density when the density reaches half filling. In Fig. 4(f), we consider $U^{\prime}<0$, using only the single-site potential since the DMFT calculation have so far been restricted to $U^{\prime}>0$. We see that the single-site potential gives very good agreement with the exact results.
[1] R. E. Peierls, Quantum Theory of Solids (Oxford University Press, Oxford, UK, 2001).

[2] G. D. Mahan, Many-Particle Physics (Springer, Berlin, 2000).

[3] O. Rösch, J. E. Han, O. Gunnarsson, and V. H. Crespi, Phys. Status Solidi B 242, 118 (2005).

[4] A. J. Leggett, Nat. Phys. 2, 134 (2006).

[5] Y. Hu, J. W. F. Venderbos, and C. L. Kane, Phys. Rev. Lett. 121, 126601 (2018).

[6] J. Hubbard, Proc. R. Soc. London A 281, 401 (1964).

[7] T. Holstein, Ann. Phys. 8, 325 (1959).

[8] J. O. Lawson, Physica A (Amsterdam, Neth.) 89, 139 (1977).

[9] J. K. Freericks and M. Jarrell, Phys. Rev. Lett. 75, 2570 (1995).

[10] F. Becca, M. Tarquini, M. Grilli, and C. Di Castro, Phys. Rev. B 54, 12443 (1996).

[11] G. S. Jeon, T.-H. Park, J. H. Han, H. C. Lee, and H.-Y. Choi, Phys. Rev. B 70, 125114 (2004).

[12] W. Koller, D. Meyer, Y. Ōno, and A. C. Hewson, Europhys. Lett. 66, 559 (2004).

[13] G. Sangiovanni, M. Capone, C. Castellani, and M. Grilli, Phys. Rev. Lett. 94, 026401 (2005).
[14] J. Bauer, Europhys. Lett. 90, 27002 (2010).

[15] Y. Murakami, P. Werner, N. Tsuji, and H. Aoki, Phys. Rev. B 88, 125126 (2013).

[16] P. Werner and M. Casula, J. Phys.: Condens. Matter 28, 383001 (2016).

[17] P. Werner and M. Eckstein, Phys. Rev. B 88, 165108 (2013).

[18] S. Sayyad, R. Žitko, H. U. R. Strand, P. Werner, and D. Golež, Phys. Rev. B 99, 045118 (2019).

[19] G. De Filippis, V. Cataudella, E. A. Nowadnick, T. P. Devereaux, A. S. Mishchenko, and N. Nagaosa, Phys. Rev. Lett. 109, 176402 (2012).

[20] P. Werner and M. Eckstein, Europhys. Lett. 109, 37002 (2015).

[21] R. Micnas, M. H. Pedersen, S. Schafroth, T. Schneider, J. J. Rodriguez-Nunez, and H. Beck, Phys. Rev. B 52, 16223 (1995).

[22] V. L. Campo Jr. and K. Capelle, Phys. Rev. A 72, 061602(R) (2005).

[23] E. Perfetto and G. Stefanucci, Phys. Rev. B 86, 081409(R) (2012).

[24] M. Capone, C. Castellani, and M. Grilli, Adv. Condens. Matter Phys. 2010, 920860 (2010). 
[25] P. Hohenberg and W. Kohn, Phys. Rev. 136, B864 (1964).

[26] W. Kohn and L. J. Sham, Phys. Rev. 140, A1133 (1965).

[27] E. Runge and E. K. U. Gross, Phys. Rev. Lett. 52, 997 (1984).

[28] See, e.g., R. O. Jones, Rev. Mod. Phys. 87, 897 (2015).

[29] S. Botti, A. Schindlmayr, R. Del Sole, and L. Reining, Rep. Prog. Phys. 70, 357 (2007).

[30] For a recent density matrix approach, see T. E. Reinhard, U. Mordovina, C. Hubig, J. S. Kretchmer, U. Schollwock, H. Appel, M. A. Sentef, and A. Rubio, J. Chem. Theory Comput. 15, 2221 (2019).

[31] T. Kreibich and E. K. U. Gross, Phys. Rev. Lett. 86, 2984 (2001).

[32] O. Butriy, H. Ebadi, P. L. de Boeij, R. van Leeuwen, and E. K. U. Gross, Phys. Rev. A 76, 052514 (2007).

[33] M. Farzanehpour and I. V. Tokatly, Phys. Rev. B 90, 195149 (2014).

[34] M. Ruggenthaler, J. Flick, C. Pellegrini, H. Appel, I. V. Tokatly, and A. Rubio, Phys. Rev. A 90, 012508 (2014).

[35] W. Metzner and D. Vollhardt, Phys. Rev. Lett. 62, 324 (1989).

[36] A. Georges, G. Kotliar, W. Krauth, and M. J. Rozenberg, Rev. Mod. Phys. 68, 13 (1996).

[37] P. Werner and A. J. Millis, Phys. Rev. Lett. 99, 146404 (2007).

[38] I. G. Lang and Yu. A. Firsov, Zh. Eksp. Teor. Fiz. 43, 1843 (1962) [Sov. Phys. JETP 16, 1301 (1963)].

[39] O. Gunnarsson and K. Schönhammer, Phys. Rev. Lett. 56, 1968 (1986).

[40] N. A. Lima, M. F. Silva, L. N. Oliveira, and K. Capelle, Phys. Rev. Lett. 90, 146402 (2003).

[41] F. Aryasetiawan, O. Gunnarsson, and A. Rubio, Europhys. Lett. 57, 683 (2002).

[42] C. Verdozzi, Phys. Rev. Lett. 101, 166401 (2008).

[43] R. Baer, J. Chem. Phys. 128, 044103 (2008).

[44] Y. Li and C. A. Ullrich, J. Chem. Phys. 129, 044105 (2008)

[45] G. Stefanucci, E. Perfetto, and M. Cini, Phys. Rev. B 81, 115446 (2010).

[46] M. Farzanehpour and I. V. Tokatly, Phys. Rev. B 86, 125130 (2012).

[47] J. I. Fuks and N. T. Maitra, Phys. Rev. A 89, 062502 (2014).

[48] M. Ruggenthaler, M. Penz, and R. van Leeuwen, J. Phys.: Condens. Matter 27, 203202 (2015).

[49] D. J. Carrascal, J. Ferrer, J. C. Smith, and K. Burke, J. Phys.: Condens. Matter 29, 019501 (2017).

[50] J. Nelson, R. Tiwari, and S. Sanvito, Phys. Rev. B 99, 075132 (2019).
[51] C. A. Custodio, E. R. Filletti, and V. V. França, Sci. Rep. 9, 1886 (2019).

[52] G. Xianlong, M. Polini, M. P. Tosi, V. L. Campo, K. Capelle, and M. Rigol, Phys. Rev. B 73, 165120 (2006).

[53] G. Xianlong, M. Rizzi, M. Polini, R. Fazio, M. P. Tosi, V. L. Campo, and K. Capelle, Phys. Rev. Lett. 98, 030404 (2007).

[54] D. Karlsson, C. Verdozzi, M. M. Odashima, and K. Capelle, Europhys. Lett. 93, 23003 (2011).

[55] A. Kartsev, D. Karlsson, A. Privitera, and C. Verdozzi, Sci. Rep. 3, 2570 (2013).

[56] G. Stefanucci and S. Kurth, Phys. Rev. Lett. 107, 216401 (2011).

[57] J. P. Bergfield, Z. F. Liu, K. Burke, and C. A. Stafford, Phys. Rev. Lett. 108, 066801 (2012).

[58] F. Evers and P. Schmitteckert, Phys. Chem. Chem. Phys. 13, 14417 (2011).

[59] S. Kurth, G. Stefanucci, E. Khosravi, C. Verdozzi, and E. K. U. Gross, Phys. Rev. Lett. 104, 236801 (2010).

[60] G. Stefanucci and S. Kurth, Nano Lett. 15, 8020 (2015).

[61] D. Karlsson, M. Hopjan, and C. Verdozzi, Phys. Rev. B 97, 125151 (2018).

[62] M. Herrera, R. M. Serra, and I. D’Amico, Sci. Rep. 7, 4655 (2017).

[63] For a review, see K. Capelle and V. L. Campo, Phys. Rep. 528, 91 (2013).

[64] K. Schönhammer, O. Gunnarsson, and R. M. Noack, Phys. Rev. B 52, 2504 (1995).

[65] D. Karlsson, A. Privitera, and C. Verdozzi, Phys. Rev. Lett. 106, 116401 (2011).

[66] D. Jacob and S. Kurth, Nano Lett. 18, 2086 (2018).

[67] P. S. Cornaglia, H. Ness, and D. R. Grempel, Phys. Rev. Lett. 93, 147201 (2004)

[68] A systematic investigation of the $U^{\prime}<0$ regime is left to future work.

[69] N. Blümer, Ph.D. thesis, Universität Augsburg, 2003.

[70] Time Dependent Density Functional Theory, edited by M. A. L. Marques, C. Ullrich, F. Nogueira, A. Rubio, K. Burke, and E. K. U. Gross (Springer-Verlag, Berlin, 2006).

[71] A. Zangwill and P. Soven, Phys. Rev. A 21, 1561 (1980).

[72] M. Levý, Proc. Natl. Acad. Sci. USA 76, 6062 (1979).

[73] C.-O. Almbladh (private communication).

[74] P. Werner, A. Comanac, L. de' Medici, M. Troyer, and A. J. Millis, Phys. Rev. Lett. 97, 076405 (2006).

[75] K. E. Cahill and R. J. Glauber, Phys. Rev. 177, 1857 (1969).

[76] D. Langreth, Phys. Rev. B 1, 471 (1970). 